\title{
HPM for Solving the Time-fractional Coupled Burger's Equations
}

Khadijah M. Abualnaja

Department of Mathematics and Statistics, College of Science, Taif University, Taif, KSA

dujam@windowslive.com

\section{ABSTRACT}

This paper is devoted to derive the approximate solutions for the time-fractional (Caputo sense) coupled Burger's equations with implementation of the homotopy perturbation method. The numerical results are compared with the exact solution. The results reveal that the presented technique is very effective and simple.

Keywords: Fractional differential equations; Caputo's derivative; Homotopy perturbation method.

\section{MSC 2010: 65N20; $41 \mathrm{A30}$.}

\section{INTRODUCTION}

In this paper, we will implement one of these methods, namely, homotopy perturbation method (HPM) ([7]-[10]), to solve the propsed problem ([1], [3], [6], [12]). Unlike the traditional numerical methods, such as, the finite element method [5] and the finite difference method [19], HPM does not need, discretization or linearization. Recently, the applications of homotopy theory among scientists appeared, and the homotopy theory becomes a powerful mathematical tool, when it is successfully coupled with perturbation theory. Many authors (see [4], [11], [15]-[17]) are pointed out that HPM can overcome the difficulties arising in calculation of Adomian's polynomials in Adomian decomposition method.

\section{Definition 1.}

The Caputo fractional derivative of the function $g(t)$ is defined as follows:

$$
D^{\alpha} g(t)=\frac{1}{\Gamma(n-\alpha)} \int_{0}^{t} \frac{g^{(n)}(\tau)}{(t-\tau)^{\alpha-n+1}} d \tau, \quad n-1<\alpha \leq n, \quad t>0 .
$$

In the following proposition, we will state a basic property.

\section{Proposition 1. [13]}

If $m-1<\alpha<m, m \in \mathrm{N}$, and $f(x) \in C_{\mu}^{m}, \mu \geq-1$, then:

$$
D^{\alpha} J^{\alpha} f(x)=f(x), \quad \text { and } \quad J^{\alpha} D^{\alpha} f(x)=f(x)-\sum_{k=0}^{m-1} f^{(k)}(0) \frac{x^{k}}{k !}, \quad x>0 .
$$

The Caputo fractional derivative operator is satisfied the linear relationship and the so-called Leibnitz rule, that's:

$$
\begin{aligned}
& D^{\alpha}\left[c_{1} f(x)+c_{2} g(x)\right]=c_{1} D^{\alpha} f(x)+c_{2} D^{\alpha} g(x) ; \\
& D^{\alpha}[f(x) g(x)]=\sum_{r=0}^{\infty}\left(\begin{array}{l}
\alpha \\
r
\end{array}\right) f^{(r)}(x) D^{\alpha-r} g(x) .
\end{aligned}
$$

where $c_{1}$ and $c_{2}$ are constants, $f(x)$ is continuous in $[a, x]$ and $g(x)$ has $n+1$ continuous derivatives in [a,x]. For the Caputo's derivative we have $D^{\alpha} C=0, \quad \mathrm{C}$ is a constant, and [18] 
(4)

For more details on fractional derivatives definitions and their properties see ([13], [18]).

\section{Approximate Solution using HPM}

In this section, we will illustrate how implement HPM to obtain the approximate solutions of the fraction non-linear coupled system of Burger's equations of the following form:

$$
\begin{array}{ll}
D^{\alpha} u-u_{x x}-2 u u_{x}+(u v)_{x}=0, & 0<\alpha \leq 1, \mathrm{t}>0, \\
D^{\beta} v-v_{x x}-2 v v_{x}+(u v)_{x}=0, & 0<\beta \leq 1,
\end{array}
$$

subject to the following initial conditions:

$$
u(x, 0)=v(x, 0)=f(x)=\sin (x) .
$$

The exact solution of (5)-(6) for the special case $\alpha=\beta=1$ is given by:

$$
u(x, t)=v(x, t)=e^{-t} \sin (x) .
$$

In fact, different response systems can be obtained when at less one of the parameters $\alpha$ and $\beta$ varies. when $\alpha=\beta=1$ the fractional equations reduce to the classical coupled Burger's equations.

Now, we will apply the HPM to obatin the approximate solutions of the system (5)-(6).

Using the HPM, we can construct the following simple homotopies:

$$
\begin{aligned}
& H_{1}(u, q)=D^{\alpha} u-q\left[u_{x x}+2 u u_{x}-(u v)_{x}\right]=0, \\
& H_{2}(v, q)=D^{\beta} v-q\left[v_{x x}+2 v v_{x}-(u v)_{x}\right]=0,
\end{aligned}
$$

where $q \in[0,1]$ is an embedding parameter. In case $q=0$, Eqs. (9)-(10) become linear equations, which are easy to solve, and when $q=1$, Eqs.(9)-(10) turn out to be the original equations (5)-(6).

Now, we will use the homotopy parameter $q$ to expand the solutions:

$$
\begin{gathered}
u(x, t)=u_{0}+q u_{1}+q^{2} u_{2}+q^{3} u_{3}+\ldots \\
v(x, t)=v_{0}+q v_{1}+q^{2} v_{2}+q^{3} v_{3}+\ldots
\end{gathered}
$$

the approximate solution can be obtained by setting $q=1$ in equations (11)-(12):

$$
u(x, t)=u_{0}+u_{1}+u_{2}+u_{3}+\ldots, \quad v(x, t)=v_{0}+v_{1}+v_{2}+v_{3}+\ldots
$$

Substituting from (11)-(12) into (5) and (6), respectively, and equating the terms with the identical powers of $q$, we can obtain a system of linear equations. Here we only write the first few linear equations: 
$D^{\alpha} u_{0}(x, t)=0$

$$
u_{0}(x, 0)=\sin (x)
$$

$D^{\beta} v_{0}(x, t)=0$

$v_{0}(x, 0)=\sin (x)$

$D^{\alpha} u_{1}(x, t)=u_{0 x x}+2 u_{0} u_{0 x}-\left(u_{0} v_{0 x}+v_{0} u_{0 x}\right)$,

$u_{1}(x, 0)=0$

$D^{\beta} v_{1}(x, t)=v_{0 x x}+2 v_{0} v_{0 x}-\left(u_{0} v_{0 x}+v_{0} u_{0 x}\right)$,

$v_{1}(x, 0)=0$,

$D^{\alpha} u_{2}(x, t)=u_{1 x x}+2\left(u_{0} u_{1 x}+u_{1} u_{0 x}\right)-\left(u_{0} v_{1 x}+u_{1} v_{0 x}+v_{0} u_{1 x}+v_{1} u_{0 x}\right)$,

$u_{2}(x, 0)=0$,

$D^{\beta} v_{2}(x, t)=v_{1 x x}+2\left(v_{0} v_{1 x}+v_{1} v_{0 x}\right)-\left(u_{0} v_{1 x}+u_{1} v_{0 x}+v_{0} u_{1 x}+v_{1} u_{0 x}\right)$,

$v_{2}(x, 0)=0$.

Take the inverse operator $J^{\alpha}$ and ${ }^{\beta}$ on both sides of the Eqs.(14)-(19), we will use the initial conditions in the following form:

$$
u_{0}(x, t)==\sin (x), \quad v_{0}(x, t)=\sin (x) .
$$

So, we can obatin the approximate solutions of the system (14)-(19) in the following form:

$$
\begin{aligned}
u_{1}(x, t) & =J^{\alpha}\left[u_{0 x x}+2 u_{0} u_{0 x}-\left(u_{0} v_{0 x}+v_{0} u_{0 x}\right]=-\sin (x) \frac{t^{\alpha}}{\Gamma(1+\alpha)},\right. \\
v_{1}(x, t) & =J^{\beta}{ }_{\left[v_{0 x x}\right.}+2 v_{0} v_{0 x}-\left(u_{0} v_{0 x}+v_{0} u_{0 x}\right]=-\sin (x) \frac{{ }_{t}^{\beta}}{\Gamma(1+\beta)}, \\
u_{2}(x, t) & =J^{\alpha}\left[u_{1 x x}+2\left(u_{0} u_{1 x}+u_{1} u_{0 x}\right)-\left(u_{0} v_{1 x}+u_{1} v_{0 x}+v_{0} u_{1 x}+v_{1} u_{0 x}\right)\right] \\
& =\sin (x)(1-3 \cos (x)) \frac{t^{2 \alpha}}{\Gamma(1+2 \alpha)}+\sin (x) \cos (x) \frac{t^{\alpha+\beta}}{\Gamma(1+\alpha+\beta)}, \\
v_{2}(x, t) & =J v^{\beta}\left[v_{1 x x}+2\left(v_{0} v_{1 x}+v_{1} v_{0 x}\right)-\left(u_{0} v_{1 x}+u_{1} v_{0 x}+v_{0} u_{1 x}+v_{1} u_{0 x}\right)\right] \\
& =\sin (x)(1-3 \cos (x)) \frac{t^{2 \beta}}{\Gamma(1+2 \beta)}+\sin (x) \cos (x) \frac{t^{\alpha+\beta}}{\Gamma(1+\alpha+\beta)}, \ldots . .
\end{aligned}
$$

Therefore, the approximate solution can be presented by the same iterative process, in the following series form:

$$
\begin{aligned}
& u(x, t)=f-f \frac{t^{\alpha}}{\Gamma(1+\alpha)}+f\left(1-3 f_{x}\right) \frac{t^{2 \alpha}}{\Gamma(1+2 \alpha)}+f f_{x} \frac{t^{\alpha+\beta}}{\Gamma(1+\alpha+\beta)}+\ldots, \\
& v(x, t)=f-f \frac{{ }_{t}^{\beta}}{\Gamma(1+\beta)}+f\left(1-3 f_{x}\right) \frac{t^{2 \beta}}{\Gamma(1+2 \beta)}+f f_{x} \frac{t^{\alpha+\beta}}{\Gamma(1+\alpha+\beta)}+\ldots
\end{aligned}
$$

Where $f=f(x)=\sin (x)$. In order to illustrate the advantages and the accuracy of HPM for solving the presented problem, we calculate the second order approximation, i.e.:

$$
u_{H P M}(x, t)=u_{0}+u_{1}+u_{2}+\ldots, \quad v_{H P M}(x, t)=v_{0}+v_{1}+v_{2}+\ldots
$$

The behavior of the approximate solution is given the figure 1 and compared with the exact solution in figure 2 .

\section{Conclusion and General Remarks}



In this article, we implemented HPM for obtaining the approximate solution of the time-fractional coupled Burger's equations. From the introduced numerical results, we can conclude the following properties of the presented method:

(1) HPM gives a simple and a powerful mathematical tool for solving the FDEs;

(2) HPM is an efficient and powerful method for solving a wide class of fractional differential equations;

(3) HPM is straightforward without any restrictive assumptions and special techniques;

(4) HPM gives the approximate solution with higher accuracy and fast convergence rate.
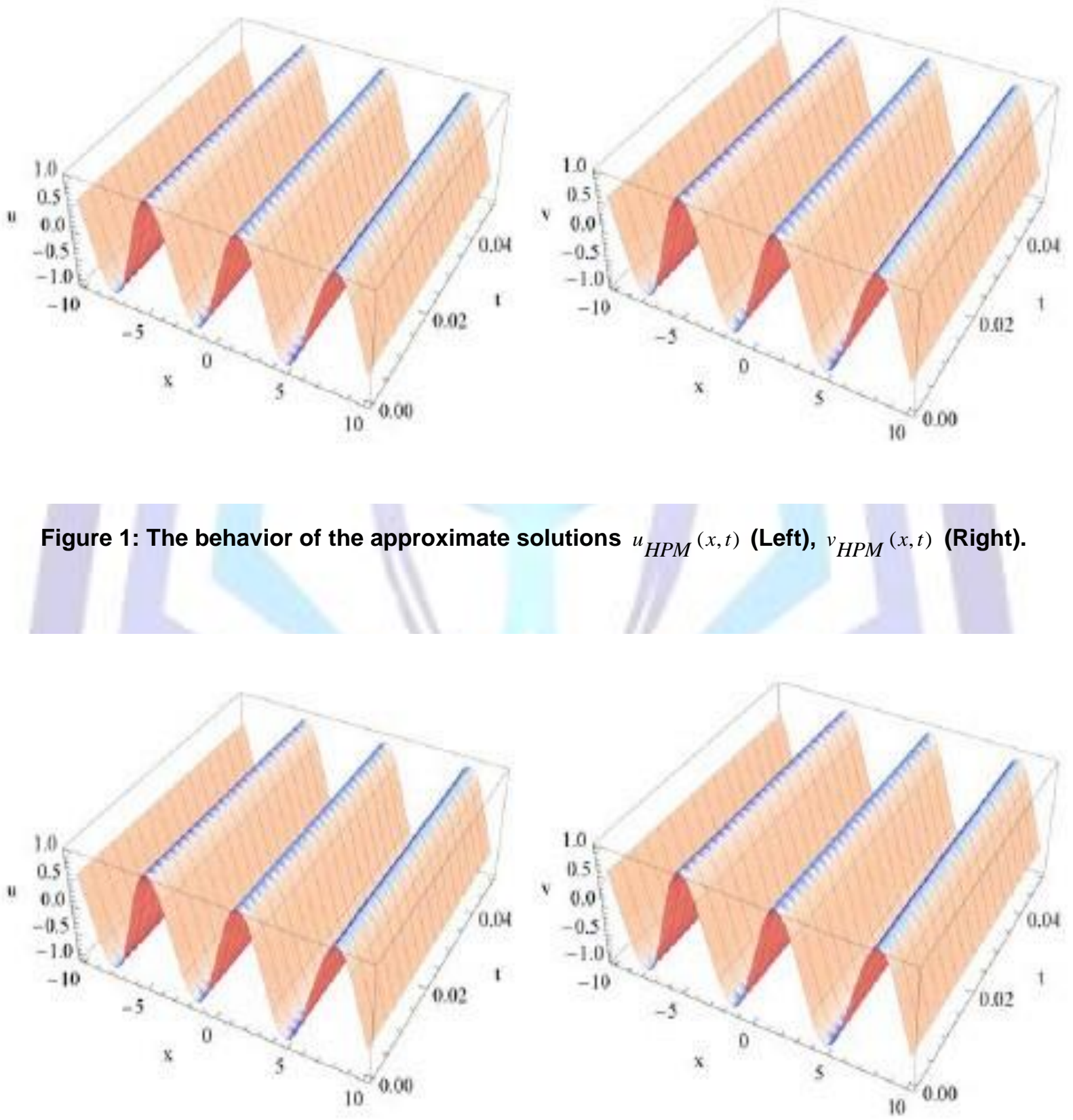

Figure 2: The behavior of the exact solution $u(x, t)$ (Left), $v(x, t)$ (Right). 


\section{ACKNOWLEDGMENTS}

The author is very grateful to the editor and the referee for carefully reading the paper and for their comments and suggestions, which have improved the paper.

\section{REFERENCES}

I. Abdou, M. A. and Soliman, A. A. 2005. Variational iteration method for solving Burger's and coupled Burger's equations. J. Comput. Appl. Math. 181, 245-251.

II. Biazar, J. and Ghazvini, H. 2009. Convergence of the HPM for partial differential equations. Nonlinear Analysis: Real World Applications 10, 2633-2640.

III. Chen, Y. and Hong-Li An, 2008. Numerical solutions of coupled Burger's equations with time- and spacefractional derivatives. Applied Mathematics and Computation, 200, 87-95.

IV. Daftardar-Gejji, V. and Jafari, H. 2007. Solving a multi-order fractional differential equation using Adomian decomposition. Applied Mathematics and Computation, 189, 541-548.

V. Enelund, M. and Josefson, B. L. 1997. Time-domain finite element analysis of viscoelastic structures with fractional derivatives constitutive relations. AIAA J., 35(10), 1630-1637.

VI. Esipov, S. E. 1995. Coupled Burger's equations: a model of polydispersive sedimentation. Phys. Rev. E, 52, 3711-3718.

VII. He, J. H. 1998. Approximate analytical solution for seepage flow with fractional derivatives in porous media. Computer Methods in Applied Mechanics and Engineering, 167(1-2), 57-68.

VIII. He, J. H. Homotopy perturbation method for solving boundary value problems. Physics Letters A, 350, 8788.

IX. He, J. H. 2005. Application of homotopy perturbation method to nonlinear wave equations. Chaos, Solitons and Fractals, 26, 695-700.

X. He, J. H. 2007. Some applications of nonlinear fractional differential equations and their approximations. Bull. Sci. Technol., 15(2), 86-90.

XI. Jafari, H. and Daftardar-Geji, V. 2006. Solving linear and nonlinear fractional diffusion and wave equations by Adomian decomposition, Appl. Math. and Comput., 180, 488-497..

XII. Podlubny, I. 1999. Fractional Differential Equations, Academic Press, New York.

XIII. Saha, S. Ray and Bera, R. K. 2005. An approximate solution of a nonlinear fractional differential equation by Adomian decomposition method. Appl. Math. Comput., 167, 561-571.

XIV. Sweilam, N. H. Khader, M. M. and Al-Bar, R. F. 2007. Numerical studies for a multi-order fractional differential equation. Physics Letters A, 371, 26-33. 
XV. Sweilam, N. H. and Khader, M. M. 2009. Exact solutions of some coupled nonlinear partial differential equations using the homotopy perturbation method. Computers and Mathematics with Applications, 58, 2134-2141.

XVI. Yuste, S. B. 2006. Weighted average finite difference methods for fractional diffusion equations. Journal of Computational Physics, 216, 264-274.

\section{Author' biography}

Khadijah Mohammed Abualnaja was born in 1974 in KSA. She received her B.S. degree from Taif University, M.S. and Ph.D degrees from King Abdulaziz University, in 1998, 2006 and 2010, respectively, and is currently Associate Professor in the Mathematics and Statistic Department of Taif University, KSA. Her main research interests are the Numerical Analysis for all kinds of equations.

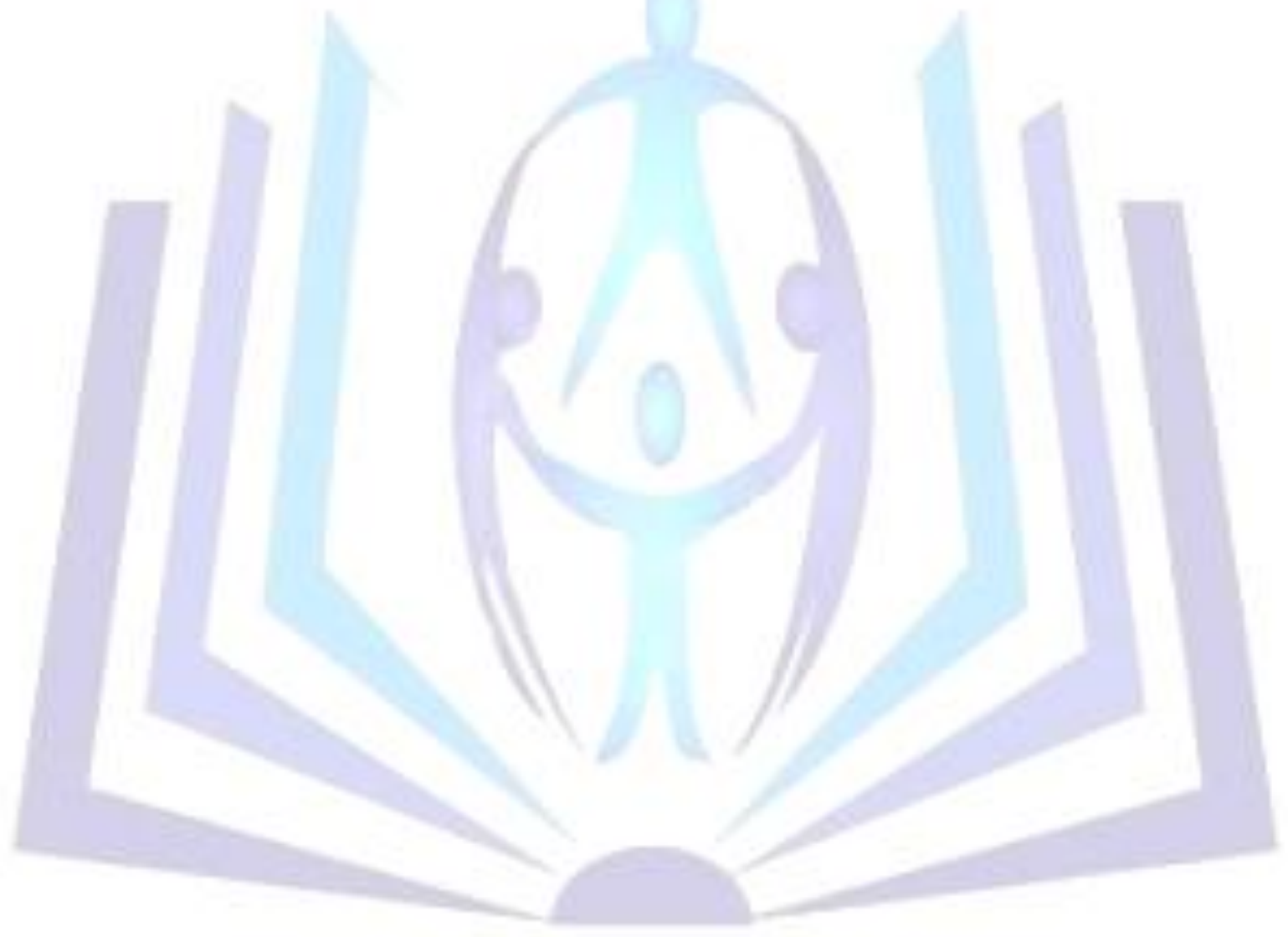

